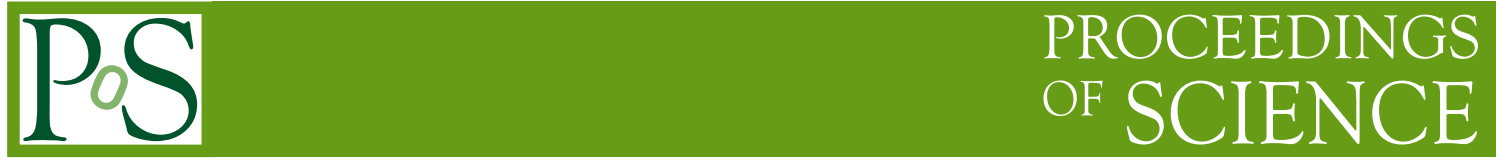

\title{
Synthesis of CNO elements in standard BBN
}

\author{
Fabio locco* \\ Università di Napoli "Federico II" \\ Dip. Scienze Fisiche, via Cintia, 80126 Napoli, Italy \\ Kavli Institute for Particle Astrophysics and Cosmology \\ PO Box 20450, MS 29, Stanford, CA 94309, USA \\ E-mail: iocco@na.infn.it

\section{Pasquale D. Serpico} \\ Max Planck Institut für Physik, (Werner Heisenberg Institut), \\ Föhringer Ring 6, 80805, München, Germany \\ E-mail: serpico@mppmu.mpg.de
}

\begin{abstract}
We perform an analysis of the $\mathrm{CNO}$ elements production in a standard big bang nucleosynthesis (BBN) scenario. The goal is to provide a sound estimate on the very low but yet poorly explored abundance of such elements (with critical importance in Population III Stars). We examine the synthesis channels for these elements in the Wagoner-Kawano code (already critically revised and updated in recent analysis); in light of the findings on CNO elements productions in BBN we add 4 nuclides and more than 200 reactions to the existing network; our results show no major discrepancies with the ones from smaller nuclear network. The robustness of the standard BBN predictions-Population III Stars were metal-free-is confirmed.
\end{abstract}

International Symposium on Nuclear Astrophysics - Nuclei in the Cosmos IX June 25-30 2006 CERN, Geneva, Switzerland

\footnotetext{
${ }^{*}$ Speaker.
} 


\section{Primordial Nucleosynthesis and Population III stars}

Standard BBN is a one-parameter theory, mostly depending on well known nuclear and particle physics processes at the keV-MeV scale. The only unknown parameter is the baryon fraction as, which actually depends on physics beyond the standard model. Nonetheless, nowadays as can be determined independently via cosmic microwave background (CMB) anisotropy studies [1]. Using the $\mathrm{CMB}$ value for $\omega_{b}$ turns $\mathrm{BBN}$ into a parameter-free (and thus highly predictive) theory, which can be used to check the internal consistency of the Standard Cosmological Model (SCM), to constrain astrophysics (e.g. ${ }^{7} \mathrm{Li}$ and ${ }^{3} \mathrm{He}$ stellar reprocessing) or for the exploration of exotic physics, as in [2]. In recent analyses like [3] the nuclear channels for the synthesis of light elements up to mass number $A=7$ have been carefully studied. The relevant reaction rates have also been updated with the newest experimental and theoretical estimates, thus reducing the uncertainties on the light element abundances. Indeed, the main uncertainty on nuclear abundances arises from those on nuclear rates; however this is true only when the reactions relevant for the synthesis of the elements of interest are included. The widely used Wagoner-Kawano code contains nuclides up to ${ }^{16} \mathrm{O}$, but no detailed analysis of the completness of the network for mass number $A>7$ is present in the literature. Missing reactions might have huge effects on the synthesis of CNO elements, although being of negligible entity for the "traditional" BBN predictions of light elements yields. Studies on the stars formed from the collapse of the very first structures in the Universe have shown that the physics of such objects is strongly dependent from the chemical composition of the cloud $[4,5]$. Namely, even relatively small amounts of Carbon, Nitrogen and Oxygen could modify either the collapse of the cloud and/or the evolution of the smallest among these stars [6]. The very low fraction of $\mathrm{CNO}$ elements necessary to modify the physics of these processes (respectively

$10^{-4}$ and $10^{-11}$ ) has motivated the current study; our purpose is to explore the synthesis channels of Carbon, Nitrogen and Oxygen in BBN and put sound constraints on their abundances by checking the consistency of the nuclear network and correctly estimating uncertainties.

\section{Current nuclear network analysis}

We have analized the synthesis channels for Carbon, Nitrogen and Oxygen in the currently used network in BBN, as critically modified for the light elements network in [3]. We have found that the small amounts of Carbon produced are synthesized via the direct reaction channel

$$
{ }^{7} \operatorname{Li}(\alpha, \gamma){ }^{11} \mathrm{~B}(p, \gamma){ }^{12} \mathrm{C}
$$

or the secondary one

$$
{ }^{7} \operatorname{Li}(\alpha, \gamma){ }^{11} \mathrm{~B}(d, n){ }^{12} \mathrm{C} .
$$

No one of these reactions is responsible for the final abundance of ${ }^{11} \mathrm{~B}$; during $\mathrm{BBN}$ they do in fact compete with the stronger ${ }^{11} \mathrm{~B}(p, \alpha) 2{ }^{4} \mathrm{He}$, which depletes all the ${ }^{11} \mathrm{~B}$. The final abundance of this element is provided by the $\beta$ decay of ${ }^{11} \mathrm{C}$ produced by $\alpha$ capture on ${ }^{7} \mathrm{Be}$, which is Coulombbarrier suppressed with respect to the analogous $\alpha$ capture on ${ }^{7} \mathrm{Li}$. The ${ }^{11} \mathrm{~B}$ left at the end of the 


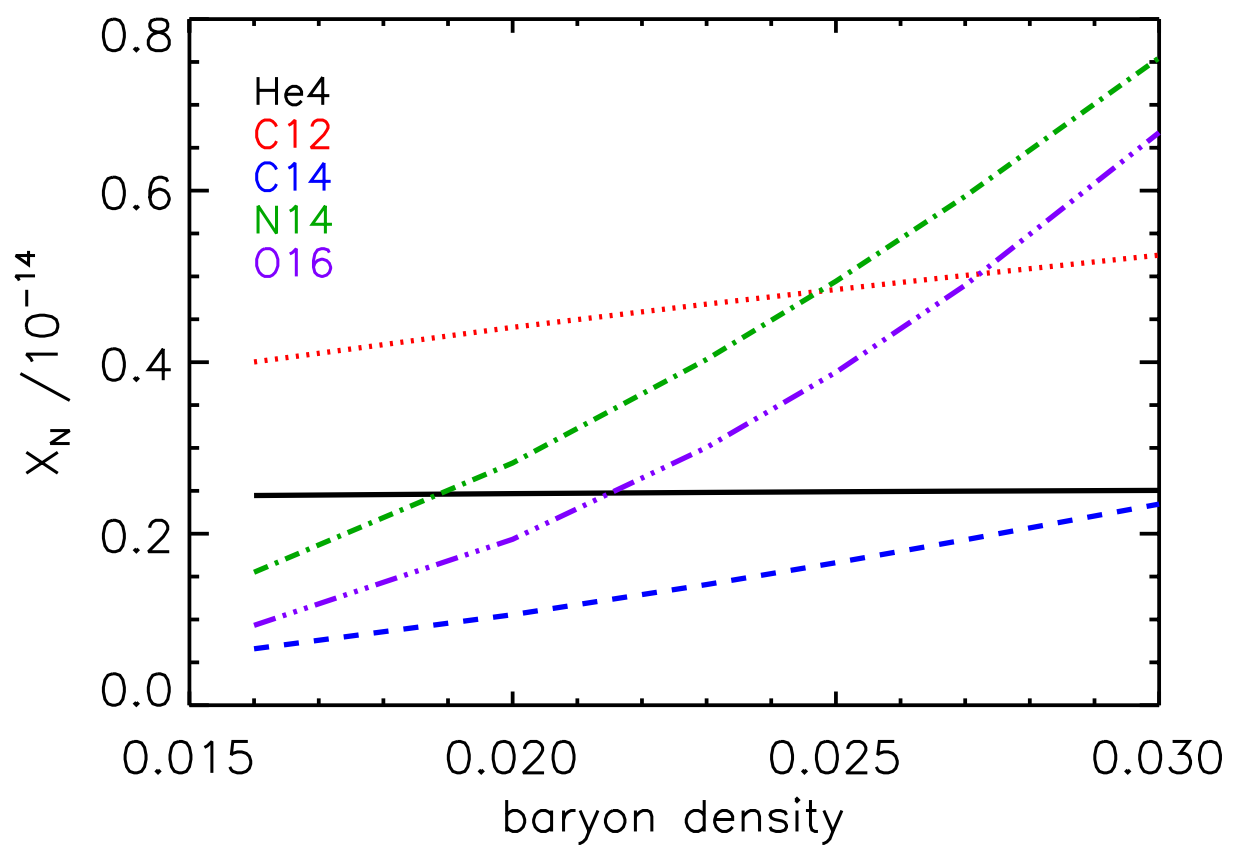

Figure 1: ${ }^{12} \mathrm{C}(\times 10),{ }^{14} \mathrm{C}\left(\times 10^{2}\right),{ }^{14} \mathrm{~N}\left(\times 10^{2}\right),{ }^{16} \mathrm{O}\left(\times 10^{5}\right)$ abundances as a function of the baryon density $\omega_{b}$ within the $1 \sigma$ interval $\omega_{b}=0.0223 \pm 0.0008$ as from [1]. The ${ }^{4} \mathrm{He}$ mass fraction $\left(\times 10^{-14}\right)$ is reported as well to show how they have the same dependence from this parameter; this is a direct consequence of the $\mathrm{CNO}$ elements production taking place mainly through the single channel ${ }^{7} \operatorname{Li}(\alpha, \gamma){ }^{11} \mathrm{~B}$.

$\mathrm{BBN}$ is therefore produced through the channel

$$
{ }^{7} \mathrm{Be}(\alpha, \gamma){ }^{11} \mathrm{C} \rightarrow{ }^{11} \mathrm{~B}
$$

Another possible path is via the unstable nuclei with $A=8$,

$$
\begin{aligned}
& { }^{8} \mathrm{Li}(\alpha, \gamma){ }^{12} \mathrm{~B} \rightarrow{ }^{12} \mathrm{C} \\
& { }^{8} \mathrm{~B}(\alpha, \gamma){ }^{12} \mathrm{~N} \rightarrow{ }^{12} \mathrm{C},
\end{aligned}
$$

where ${ }^{8} \mathrm{Li}$ is produced e.g. via ${ }^{7} \mathrm{Li}(n, \gamma)$ and ${ }^{6} \mathrm{Li}(t, p)$, and ${ }^{8} \mathrm{~B}$ via ${ }^{7} \mathrm{Be}(p, \gamma)$. According to our estimates, however, these channels only provide a sub-leading contribution to the $\mathrm{CNO}$ abundances.

The production of heavier isotopes of Carbon, Nitrogen and Oxygen proceeds by radiative proton capture on carbon nuclei, followed by a $(n, p)$ reaction. For example, ${ }^{13} \mathrm{C}$ is produced along the following channel from light elements

$$
{ }^{7} \operatorname{Li}(\alpha, \gamma){ }^{11} \mathrm{~B}(p, \gamma){ }^{12} \mathrm{C}(p, \gamma){ }^{13} \mathrm{~N}(n, p){ }^{13} \mathrm{C}
$$

and ${ }^{14} \mathrm{C}$ proceeds along from ${ }^{13} \mathrm{C}$ according to the same path

$$
{ }^{13} \mathrm{C}(p, \gamma){ }^{14} \mathrm{~N}(n, p){ }^{14} \mathrm{C} .
$$


Nitrogen is produced by means of proton radiative capture on Carbon nuclei-e.g. ${ }^{12} \mathrm{C}(p, \gamma){ }^{13} \mathrm{~N}-$ and heavier isotopes like Oxygen ones are produced with the same mechanism illustrated so far. Note that once heavier elements $(A \geq 12)$ have been synthesized, they are not disrupted back into light elements. It is worth noticing how the mechanism for ${ }^{12} \mathrm{C}$ production is completely different from the well known $3 \alpha$ process taking place in stars. This is easily explained in terms of $(i)$ the very low density of the plasma during BBN (less than $10^{20}$ baryons $/ \mathrm{cm}^{3}$ at $T \lesssim 100 \mathrm{keV}$ ); (ii) the Coulomb suppression relevant for heavier nuclei; (iii) the short time-scales involved, of the order of minutes. Synthesis of CNO elements in BBN does hence proceed along a path involving intermediate mass nuclides $(4<A<12)$; it is therefore conceivable that relevant channels may have been omitted when neglecting intermediate mass elements.

\section{Updating the BBN code}

We have modified the code presented and used in [3] adding 4 nuclides and more than 200 nuclear reactions previously neglected. The criterion adopted for adding new nuclides is based on their half-lives $t_{1 / 2}$ 's; we have found that 4 nuclides previously neglected in the Kawano code have $t_{1 / 2}$ 's comparable or longer than the typical times involved in BBN. We have therefore included ${ }^{9} \mathrm{Li}\left(t_{1 / 2}=0.178 \mathrm{~s}\right),{ }^{10} \mathrm{Be}\left(t_{1 / 2}=1.5 \times 10^{6}\right.$ years $),{ }^{9} \mathrm{C}\left(t_{1 / 2}=0.125 \mathrm{~s}\right),{ }^{10} \mathrm{C}\left(t_{1 / 2}=19.29 \mathrm{~s}\right)$. By adding the main reactions involving these nuclides we are including all viable "intermediate" channel connecting lighter to heavier elements. We have added reactions for these nuclides mimicking the structure of the pre-existing nuclear network, and updating where necessary. The reactions included for these nuclides (and the ones added for the nuclides already present in the code) have been selected on basic physical arguments, first of all Coulomb barrier considerations: Reactions with $Z_{1} Z_{2} \geq 12$ have not been considered as they are mostly suppressed in the late times of BBN, when metals are mainly formed, and energy is very low, namely few $\mathrm{keV}$. For each nuclide a full network including radiative capture, stripping/pick-up and charge-exchange up to ${ }^{3} \mathrm{He}$ has been implemented. For example, a list of these reactions for the neutron case is shown below

- radiative capture: $(n, \gamma) ;(p, \gamma) ;(d, \gamma)$

- neutron stripping: $(n, d) ;\left(n,{ }^{3} \mathrm{He}\right)$

- ${ }^{2} \mathrm{H}$ pickup: $(d, p) ;(d, n)$

- charge exchange: $(p, n) ;(p, n) ;\left({ }^{3} \mathrm{He}, t\right) ;\left(t,{ }^{3} \mathrm{He}\right)$.

Many of these reactions were missing in the original nuclear network of the Wagoner-Kawano code; in fact they do not have appreciable effects on the light nuclide abundances, which was the principal goal of the original codes. To evaluate the missing reaction rates, extensive use has been made of complilations and on-line libraries like [7, 8, 9]. The nuclear rates for many of these reactions, where measurements or theoretical estimates were missing, have been estimated with simple nuclear models, of the kind presented in [10]. An order of magnitude uncertainty has been assumed. After adding these reactions we have re-analized the synthesis channels for $\mathrm{C}, \mathrm{N}$, and $\mathrm{O}$. We can summarize the main features of our analysis as follows: 


\begin{tabular}{|c||c|c|c|c|c|c|c|}
\hline Nuclide & $\begin{array}{c}{ }^{9} \mathrm{Be} / \mathrm{H} \\
\left(\times 10^{-19}\right)\end{array}$ & $\begin{array}{c}{ }^{11} \mathrm{~B} / \mathrm{H} \\
\left(\times 10^{-16}\right)\end{array}$ & $\begin{array}{c}{ }^{12} \mathrm{C} / \mathrm{H} \\
\left(\times 10^{-16}\right)\end{array}$ & $\begin{array}{c}{ }^{13} \mathrm{C} / \mathrm{H} \\
\left(\times 10^{-17}\right)\end{array}$ & $\begin{array}{c}{ }^{14} \mathrm{C} / \mathrm{H} \\
\left(\times 10^{-17}\right)\end{array}$ & $\begin{array}{c}{ }^{14} \mathrm{~N} / \mathrm{H} \\
\left(\times 10^{-17}\right)\end{array}$ & $\begin{array}{c}{ }^{16} \mathrm{O} / \mathrm{H} \\
\left(\times 10^{-20}\right)\end{array}$ \\
\hline \hline Original & 2.4 & 3.9 & 4.4 & 7.6 & 0.6 & 2.6 & 1.8 \\
\hline Updated & 2.5 & 3.9 & 4.6 & 9.0 & 1.3 & 3.7 & 2.7 \\
\hline
\end{tabular}

Table 1: Some "heavy" nuclei abundances (for $\omega_{b}=0.023$ ) predicted respectively by the code in [3] and by using the upper limits to the production rates in the new version described in the text.

- identification of relevant channels for CNO synthesis in the existing code

- update of the rates of relevant reactions already present

- addition of 4 nuclides: ${ }^{9} \mathrm{Li},{ }^{10} \mathrm{~B},{ }^{9} \mathrm{C},{ }^{10} \mathrm{C}$

- addition of more than 200 nuclear reactions previously neglected

As it can be seen from Tab. 1, no major change is found when enlarging the network despite using upper limits for the rates in the new updated version of the code. The physical reason is that at the beginning of the BBN, when the higher temperature and density would favor the formation of CNO elements, their synthesis is suppressed by the high entropy and the lack of necessary intermediate elements. On the other hand, when enough "catalyzers" are produced at late times temperature and density are not high enough to favor the reactions by overcoming the high Coulomb barrier. Of course, all the newly introduced nuclei take part to the nucleosynthesis, but they decay or are processed into heavier ones by the end of BBN.

Even taking into account a much enlarged network, one might still wonder how robust are previous estimates, especially given the lack of data or detailed theoretical models for several reactions. To obtain a generous but more robust upper limit to the abundance of Carbon, Nitrogen, and Oxygen in BBN, we have performed an analysis of the final abundances of these elements assuming that all the reactions producing at some stage an element with $A>7$ "instantaneously" produce ${ }^{12} \mathrm{C}$. Namely, the yield of the $A>7$-product is formally considered as a CNO nucleus. In this way, we neglect any destruction mechanism (since CNO elements are more stable, they act as a monothonically growing reservoir), the multistep nature of the synthesis of CNO nuclides, and maximize the time available for their synthesis. As a result, we get $\mathrm{CNO} / \mathrm{H}=\leq 4 \times 10^{-9}$. This bound only depends on thermodynamical properties of the plasma at the BBN conditions, and on the well-known nuclear rates involving nuclei with $A \leq 7$.

\section{Conclusions}

Apart for predicting detailed values for the abundances of the light nuclei ${ }^{2} \mathrm{H}$ and ${ }^{3} \mathrm{He},{ }^{4} \mathrm{He}$, and ${ }^{7} \mathrm{Li}$, standard BBN also predicts that the first collapsed objects in the Universe should have no appreciable "metallicity". This prediction is crucial for determining the physical mechanisms regulating the collapse of the clouds leading to the PopIII stars, and the evolution of the smallest among these pristine stars.

Given the importance of this topic, and the incompleteness of standard BBN nuclear networks in the mass range $A>7$, we have performed a detailed study of the synthesis channels of Carbon, 
Nitrogen and Oxygen in Primordial Nucleosynthesis. We have found that Carbon is produced in a way consistently different from the usual $3 \alpha$ reaction in stars. The synthesis of ${ }^{12} \mathrm{C}$ proceeds along paths involving intermediate mass elements, namely ${ }^{11} \mathrm{~B}$ and ${ }^{11} \mathrm{C}$. The very light traces of Nitrogen and Oxygen are produced by radiative capture upon ${ }^{12} \mathrm{C}$. We have added to the code 4 nuclides and more than 200 reactions observing no sensible increase of CNO elements. Re-analysing the synthesis of CNO after the addition of the new reactions we find that the main channels for their production stay the same as before and none of the newly added reactions/nuclides opens effective channels from light to heavy elements in BBN. We regard this as a final and sound proof that only negligible traces of $\mathrm{CNO}$ elements are produced in standard BBN, in agreement with earlier and less accurate analyses. This should be regarded as a further observational test for standard BBN, since alternative theories like Inhomogeneous BBN might lead to significantly different conclusions (see e.g. [11] and Refs. therein).

\section{References}

[1] D. N. Spergel et al., "Wilkinson Microwave Anisotropy Probe (WMAP) three year results: Implications for cosmology," astro-ph/0603449.

[2] A. Cuoco, F. Iocco, G. Mangano, G. Miele, O. Pisanti and P. D. Serpico, "Present status of primordial nucleosynthesis after WMAP: results from a new BBN code," Int. J. Mod. Phys. A 19, 4431 (2004) [astro-ph/0307213].

[3] P. D. Serpico, S. Esposito, F. Iocco, G. Mangano, G. Miele and O. Pisanti, "Nuclear Reaction Network for Primordial Nucleosynthesis: a detailed analysis of rates, uncertainties and light nuclei yields," JCAP 0412, 010 (2004) [astro-ph/0408076].

[4] F. Nakamura and M. Umemura, "On the Initial Mass Function of PopulationIII Stars," ApJ 548 (2001), 19N [astro-ph/0010464].

[5] T. Abel, G. L. Bryan and M. L. Norman, "The Formation of the First Star in the Universe,", Science 295, 93 (2002) [astro-ph/0112088].

[6] I. Dominguez, O. Straniero, M. Limongi and A. Chieffi, "Do zero metal intermediate mass stars experience thermal pulses?," astro-ph/9912425.

[7] Audi et al., "The Nubase evaluation of nuclear and decay properties," Nuc. Phys.A 624 (1997), 1.

[8] Table of Isotopes, 8th edition, online at http: //www.tunl. duke. edu/nucldata.

[9] See the URL http: //www. nndc.bnl.gov/exfor3/endf00.htm.

[10] R. Wagoner, W. Fowler, F. Hoyle, "On the Synthesis of Elements at Very High Temperatures," ApJ 148 (1967), 3W.

[11] K. Jedamzik, "Did the Universe start at Zero Metallicity?," astro-ph/9911242. 